\title{
Lipopritein (a), a principle culprit: Relevance to pathobiology of hypertension, ischemic strokes and implications for novel therapeutic targets, a new puzzle found
}

\author{
Okom Nkili F. C. OFODILE, \\ Center for Cardiovascular Research (CDR), Institut für Pharmakologie, Charité - Universitätsmedizin, Berlin, Hessische Str. 3-4, 10115 Berlin, Germany
}

\begin{abstract}
Cardiometabolic and cerebrovascular disorders belong to public health problem in most industralized countries and these disorders are rapidly achieving this dubious distinction in the developing countries, as well. Efforts to arrest these disorders have today appeared unsatisfactory, for the mechanisms that underlie the disorders have not been completely resolved. However, it is now widely appreciated that high levels of Lipoprotein (a) play central role in the development of the disorders. Efforts to unravel the core of the mechanisms that underlie the above mentioned disorders has come to conclude that sthe imbalance between physiological serum levels of a macrophage secretory glycoprotein that belongs to the mammalian chitinase-like protein family, which they used to call Human cartilage glycoprotein 39(HC-gp39) and upregulated expression of $\mathrm{HC}$-gp39 may represent an important factor to explain the pathogenesis of a range of human disorders. In concord, several lines of evidence have accrued consolidating the concept that HC-gp39 plays critically important role in the pathogenesis of cardiometabolic and cerebrovascular disorders. Additionally, in commensurate to this, recently accumulating body of evidence suggests that HC-gp39 may interact with Liporotein (a), working, in concert, very probably, synergistically, and additionally in driving the pathogenesis of a range of human disorders characterized by inflammation and tissue remodeling, including cardiometabolic disorders and cerebrovascular disorders. However, the mechanism(s) underlying this interaction is presently rudimentary. In this direction, the mere fact that sound biology, biochemistry, and, as such, also, the real nature of the pathophysiology of these two organs: the heart and the brain, are without doubt vital for the human life, and, coupled with the fact that there are presently no drugs at hand to either prevent nor markedly and/or completely arrest the progression of these disorders, underscores the urgency and importance to proactively address this subject.
\end{abstract}

\section{Introduction}

It is gradually getting generally appreciated that Lipoprotein (a) associates itself with a plethora of macrophage-derived molecules in the pathogenesis of cardiometabolic and that of cerebrovascular disorders, as well. The findings that exaggerated levels of Hc-gp39 correlate strictly with the severity of disease processes in cardiometabolic disorders and other human disorders, coupled with the wide appreciation that an adverse relationship links insulin resistance, high serum levels of HCgp39 and metabolic and physiologic response of different structures of the heart and blood vessels, paved the way to the concept that Lipoprotein (a) may interact with human cartilage glycoprotein 39, working in concert, impact profoundly on the natural course of disease process (es) in cardiometabolic -and cerebrovascular pathologies. However, the mechanism(s) underlying this event remains largely undefined. Now, emerging discoveries reveal that inflammatory cascades may interact with innate immune metabolism and drive this process. It is, thus, the aim of this article to extend on this notion and discuss briefly the direct consequence(s) of the effects.

Lipoprotein (a) may interact with Human Cartilage Glycoprotein 39 (HC-gp39) and drive the disease pathogenesis: Evolving Importance in cardiometabolic and cerebrovascular medicine

Cerebrocardiovascular disease (CCVD) is one of the most common causes of morbidity and death worldwide, and, hence, represents an important public health burden. Traditional risk factors in form of family history, High blood pressure, hypercholesterolemia, diabetes mellitus and excessive smoking cannot, indeed, account for the entire risk for atherosclerotic lesions. A plethora of other potential risk factors have been discovered in an effort to improve risk assessment for atherosclerotic events. Direct compelling evidence collected from a large body of literature indicates that high plasma levels of lipoprotein (a); Lp (a) (>25 mg/dl) are potent, independent risk factor for a variety of cerebrocardiovascular diseases, including coronary heart disease (CHD), ischemic stroke, and venous thrombosis [13]. The ability of lipoprotein (a) to induce vascular inflammation, endothelial dysfunction, and vascular calcification is believed to be a common link among these disorders. Another phenomenon in this lesion is autophagy: Autophagy, a regulatable cell repair-and housekeeping (cleansing) mechanism, which is active during fasting,

Correspondence to: Okom Nkili F. C. OFODILE, PhD, Center for Cardiovascular Research, Charite Campus Mitte, Institute of Pharmacology, AG: THEURING, Charite Universitätsmedizin Berlin, N.F. Strasse 3-4, 10115 Berlin, Germany, Tel: +491747554102; Fax:+4930450525002;E-mail: ofodile120@yahoo.co.uk

Key words: inflammation, cell death, immunity, atherosclerosis, lipoprotein (a), human cartilage glycoprotein 39 (hc-gp39)

Received: May 11, 2015; Accepted: May 29, 2015; Published: June 04, 2015 
OFODILE ONFC (2015) Lipopritein (a), a principle culprit: Relevance to pathobiology of hypertension, ischemic strokes and implications for novel therapeutic targets, a new puzzle found

represents major homeostatic process, which relies on the digestion of potentially harmful intracellular structures, and or organelles, notably mitochondria, endoplasmic reticulum, and fat droplets [4-7].

Autophagy participates in a wide range of biological processes, including development, aging, degenerative disease, infectious disease and cancer. Defective autophagy may contribute in significant ways to cell death. Lipoprotein (a), a hydrophilic glycoprotein, is a modified LDL-like lipoprotein in which a Kringle-containing protein called apollipoprotein (a) is covalently bound to apolipoprotein B of LDL(Low density lipoprotein) (Koschinky and Marcovina). Lp (a) has also been identified as a carrier of oxidized phospholipids OxPLs; they are highly inflammatory, prothrombotic, provoke disordered activation of endothelial cells and quench nitric oxide. Of immense importance, Lp (a) has recently been shown to be strongly linked genetically to cardiovascular disease, and that individuals of African descent are over proportionally associated with high plasma levels of Lp (a). Emerging accumulating evidence indicates that $\mathrm{Lp}$ (a) is specifically intimately involved in advanced progression of atherosclerotic lesions. A set of experiments demonstrated that OxPL-containing Lp (a) and apolipoprotein (a) play pivotal role in triggering a chain of events that result in the development of advanced atherosclerotic lesions. In this line, many lines of evidence have shown that oxidation of low density lipoprotein (LDL) and phospholipids OxPLs is a major pathogenic factor in the development of atherosclerosis. Highly oxidized LDL (OxLDL) and OxPLs accumulate in atherosclerotic lesions and activate macrophages and other vascular cells: A central event that results in chronic inflammation in vascular wall, making atherosclerotic plagues profoundly vulnerable to rapture, and, leading to a variety of acute cardiovascular events.

An important mechanism underlying this event is attributed to the ability of OxPL-containing Lp (a) and apo (a) to trigger cell death (apoptosis) in endoplasmic reticulum-stressed macrophages in atherosclerotic lesions via pathways, including oxidative stress, and TLR-NADPH oxidase pathways. In addition, recently accumulating evidence has associated deregulated autophagy process with a plethora of OxLDL-mediated actions, thereby suggesting that regulated mitochondrial and calcium physiology play important role in this setting. Of note, emerging discoveries have come to indicate that an evolutionarily conserved macrophage-derived secretory protein they use to call human cartilage glycoprotein 39(HC-gh39)/(or YKL-40) $[8,9]$ and, lipoprotein (a), both important risk factors for endothelial dysfunction and unifying factors in the development of atherosclerotic lesions, may interact and amplify each other in a vicious circle of toxicity, leading to inflammation, excessive oxidative stress, and cellular dysfunction and eventually to cell death. HC-gp39 was first reported as a secretory glycoprotein found in the inflammed joint of arthritis patients only $[10,11]$.

In the contrary, however, HC-gp39 is presently known to be expressed and secreted in a wide variety of cells and tissues, and it is widely appreciated that HC-gp39 is associated with almost every human disorder, as well. In concord, large quantities of HC-gp39 are found in human atherosclerotic plaques. Its serum levels directly correlate with cardiovascular morbidity, and mortality and all-cause mortality; unstable angina, left ventricular dysfunction, hypertension, obesity and several types of cancer $[12,13]$. HC-gp39, an inflammationassociated protein and a regulator of innate and adaptive immunity, is a Th2-Cells responsive mammalian chitinase-like protein, and heparin, collagen -and Chitin-binding C-Lectin [14]. It works in synergism with IGF-1 and related proteins. It also exhibits biphasic dose response: a clear signature of hormesis mechanism (Okom Ofodile, 2004, unpublished.). Hormesis could be defined as a dose-response relationship phenomenon characterized by low-dose stimulation and high dose inhibition: it has been frequently observed in properly designed studies and is broadly generalizable as being independent of chemical/physical agent, biological model, and endpoint measured: EJ Calabrese and LA Baldwin [15].

The biological and clinical role of HC-gp39 is not completely defined. However, emerging investigations have provided new understanding of how HC-gp39 alterations may impact on hypertension and have results in exacerbation of both cardiovascular and cerebrovascular pathologies. High serum levels of HC-gp39, besides inducing VSMC differentiation to osteoblast-like cells, eliciting marked tissue remodeling, may induce sustained inappropriate upregulation of matrixmetalloproteinase 9/ and, possibly, 2, as well. This will lead to degradation of elastin and enhanced collagen deposition, resulting, thus, in disruption of vascular wall integrity and vascular stiffness, (for elastin plays principal role in vascular elasticity, and collagen fibers are more than 1000 times stiffer than elastin): all the the se may bear particular relevance to hypertension.

Exaggerated quantities of HC-gp39 could result in prolonged inappropriate activation of ERK/ 1 and 2 (Extracellular regulated kinase 1and 2) pathway leading to glutamate and ischemia-induced oxidative stress in neuronal cultures and brain. In almost direct association with this: because Brain-derived neurotrophic factors is very vital for the organs such as the heart and the brain, exaggerated serum levels of HCgp39 resulting in disordered expression and/or production of matrix metalloproteinases, may, in turn, potently impair the synthesis and the activity of the Brain-derived neurotrophic factors: an event that might markedly compromise insulin sensitivity and cardiovascular health, suggesting a link between HC-gp39 activity, food intake, energy expenditure and/or metabolism. Furthermore, recent hypothesis reveals that HC-gp39 may exert its biological effects through five to six mechanisms, whereby its ability to impact upon, and modulate the cell death machinery, and, also induce upregulation of angiogenic process, and, possibly, also sustaining angiogenesis may represent the major component of these mechanisms. In this context, the putative ability of HC-gp39 to modulate autophagy machinery (Okom Ofodile, 2010, Abstract. AJPP) should be associated with significant effects on human biology, including mitochondrial physiology. This is particularly relevant to degenerative diseases in which inflammation and oxidative stress occur over time, and dysfunction in both mitochondrial and autophagy pathways play a role. Another important event in this scenario may be the process of efferocytosis.

Efferocytosis is a vital cell death machinery-associated process and it is pivotal for the vascular physiology. Hence, as the central event in the development of atherosclerotic lesions is the transformation of arterial wall macrophages into lipid-laden foam cells, and, also, because, efficient clearance of endoplasmic reticulum-mediated apoptotic macrophages may modify development of advanced atherothrombotic vascular disease susceptibility, and, therefore, without doubt, high serum levels of HC-gp39, leading to perturbation of autophagic process may contribute to defective efferocytosis: an event that may markedly exacerbate advanced atherosclerotic pathology, with disastrous consequences [16,17]. Furthermore, high levels of ROS as direct consequence of perturbed mitochondrial physiology, among other things, can cause reduced myocardial contractility and lipid peroxidation of cellular membranes leading to myocyte structural damage and eventually to destabilization of atherosclerotic plaque. 
OFODILE ONFC (2015) Lipopritein (a), a principle culprit: Relevance to pathobiology of hypertension, ischemic strokes and implications for novel therapeutic targets, a new puzzle found

Extending on and, going beyond this, exaggerated serum levels of HC-gp39 leading to modulation of cell death machinery including autophagic process could, in an intricate chains of events, contribute to perturbation of the desmin architecture resulting in deleterious cellular dysfunction and, eventually sudden cardiac death [18].

Furthermore, there is possibility that HC-gp39 could interact with Fibroblast Growth factor 23 and, in turn, impacts upon the activity of Klotho protein, leading to modulation of aging processes -particularly, via aberrant tissue remodeling whereby the intricate interactions, and activities of both TGF-beta 1 and Angiotensin II may play a role in this scenario. These, in a chain of events, and altogether, may bear enormous relevance to cardiovascular health and cerebrovascular pathology, as well. This pieces of notion, based on the presently available data, might point to a role for HC-gp39 in the complicated complex machinery of human aging. (Okom Ofodile, Hypothesis: 2015, submitted).

Based on the above considerations, particularly in association with the process of autophagy, the strict correlation of the serum levels of HC-gp39 with both the normal course of disease progression and the severity of disease activity in cystic fibrosis and Inflammatory Bowel Diseases, respectively, for instance, may reflect the or an interplay between HC-gp39 and autophagic process, as HC-gp39 serum levels, in line with a large body of literature, have been repeatedly reported to be particularly upregulated in these disease states. Of note, it is fair to say that to our knowledge, this is the first serious attempt, robustly arguing in favor for an intricate interactions between Lipoprotein (a) and HC-gp39,: working, in concert, in synergy, and additionally, in driving cardiometabolic and cerebrovascular pathologies. These considerations raise the possibility that measures designed to contain the havoc-associated with high serum levels of HC-gp39 may also equally be efficacious in tackling the deleterious effects mediated by high serum levels of Lipoprotein (a) in the development and resolution of atherosclerotic lesion.

In this context, the dual role of HC-gp39 in the context of its ability to display biphasic dose response : cytoprotective at low dose and deleterious at high dose, is one of the most fascinating features of this glycoprotein, and one that needs to be understood, if we ever anticipate to harness HC-gp39 for therapeutic use. In addition to this, the multiplicity of HC-gp39 and its virtually ubiquitous expression calls for more rigorous investigation of its role in health and disease states, and more importantly, cautious evaluation of its potential as immunopharmacologic agent. In this continuum, several studies have demonstrated the ability of lipid apheresis methodology to markedly suppress the devastating health burden associated with exaggerated quantities of Lipoprotein (a): it potentially inhibit multiple components of inflammation, including complement fragments, proinflammatory cytokine production, and leucocyte cell adhesion. All this is very beneficial for the heart and the brain. Hence combined therapy involving, humanized anti- HC-gp39 antibody, complementinhibitors, endoplasmic reticulum stress inhibitor, autophagy enhancer, matrix metalloproteinase 9/2 inhibitor, TLR 9 inhibitor (to counteract possible havoc in association with escaped mitochondrial DNA) and appropriate Lipid Apheresis would be highly recommended. Given the absence of any single treatment option in a position to significantly contain the havoc directly related with high serum levels of Lipoprotein (a) and the favorable side effects characteristic of Lipid Apheresis methodology, Lipid Apheresis may be considered as the major therapy for individuals with chronic cardiovascular disorders associated with disordered Lipoprotein (a) production. In addition to the above mentioned measures, mild physical activity, occasional intermittent fasting, and consequent implementation of calorie restriction measures will be very beneficial.

Moreover, given the broad cytoprotective properties of the heat shock response, for instance, there is now strong interest in discovering and developing pharmacological agents capable of inducing the heat response. This may open new perspectives in medicine, as molecules inducing this defense mechanism appear to be possible candidates for novel cytoprotective strategies. Because mitochondrial dysfunction contributes to the pathogenesis of various cardiometabolic and neurodegenerative disorders accompanied by pathophysiological consequences at the level of calcium-driven excitotoxicity and inflammation, and, because under this condition free radical-induced injury may be a major component of calciuminduced injury, as well as macrophage mediated inflammation, early and appropriate administration of various antioxidants may be very beneficial. In this context, additional measures may be warranted. In particular, manipulation of endogenous cellular defense mechanisms, such as the heat shock response, through nutritional antioxidants, pharmacological compounds or gene transduction, may represent an innovative approach to therapeutic intervention in diseases characterized by disordered lipid metabolism, deregulated inflammatory responses (leading to chronic inflammation), excessive tissue remodeling and, defective autophagy, such as cardiometabolic disorders, cerebrovascular disorders and neurodegenerative diseases, in general.

\section{Calorie Restriction (Dietary calorie restriction)}

Calorie restriction is the most robust and effective measure in retarding the aging process [19-22]. Dietary calorie restriction (DR) also referred as Calorie Restriction (CR) is the reduction of diet consumed to a level less than that eaten by ad libidum (AL)-fed animals, without any observable malnutrition. It is now established that reduction of macronutrient can influence disease [23,24]. Calorie restriction has been reported to decrease ROS production in rat heart mitochondria by decreasing the degree of the complex I ROS generator in normal conditions [25]. Of note, a very important finding is the fact that Dietary calorie restriction is the only intervention to slow down the aging process, reverse the effect of aging of protein process, mostly through the process of autophagy. The above pieces of notion makes a case suggesting that HC-gp39 may, possibly, represent a node, linking human disorder (disease) via heat shock protein: Calorie Restriction; Autophagy and Hormesis to human health. We term this construction "ONIT-AXIS". This, in turn, implies and, at the same time, warrants us to propound that HC-gp39 may reside at the crossroads (-and/or intimately interacts with the networks) governing the cells' decision to proliferate or degenerate. The emergence of "Onit-Axis" might be evidenced by this consideration: because the pathways governing the activity of HC-gp39 largely overlap with the pathways intimatedly implicated in mediating the biological effects of calorie restriction ; namely insulin -like growth factor-1 (IGF-1),/insulin signaling pathway, the sirtuin pathway, the adenosin-monophosphate (AMP) activated protein kinase (AMPK) pathway-and a pathway very closely related to, but, not totally identical, with the mammalian target of rapamycin (mTOR) pathway, thereby pointing to a link for HCgp39, connecting diet, obesity and aging to inflammation, diabetes and related traits, and a range of other human disorders. This further suggests that the activity of HC-gp39 may be involved in the genesis and consequently, also, in the clinical outcome in almost all human disorders largely characterized by inflammation, exaggerated tissue remodeling, and defective autophagy. (Okom Ofodile, 2015). In this 
OFODILE ONFC (2015) Lipopritein (a), a principle culprit: Relevance to pathobiology of hypertension, ischemic strokes and implications for novel therapeutic targets, a new puzzle found

line, and, in commensurate to this, and, because of the central role HCgp39 plays in the context of NF-kappa B and PI(3)/AK T activation and innate immunity, Humans carrying HC-gp39 gene variation may be prone to microbial infections, autoimmune disorders and a range of other disorders characterized by deregulated inflammatory responses, and endothelial dysfunction.

\section{Conclusion}

It is essential to continue basic research on the mechanisms that mediate the biological effect(s) of HC-gp39 by exploiting technological advances and developing better animal models to validate established and newly identified pathways associated with the activity of human cartilage glycoprotein 39 (Hc-gp39). Hence, those Scientists and Clinicians who discovered HC-gp39 and, for those who have subsequently dedicated their lives' work to elucidation and targeting the nature of the biochemical effects of HC-gp39, successful translation of these effects into the clinics in the form of FDA-approved medicines will be a crowning achievement. In combination, fuller understanding of the networks governing the interaction between HC-gp39 (the molecular events governing the interaction between HC-gp39 and autophagic signaling, on the one side, and inflammatory responses, on the other hand,) and Lipoprotein (a) in the development and the resolution of cardiometabolic-and cerebrovascular pathologies may open a new dimension in this province of research which may bear profound relevance to novel therapy.

\section{Acknowledgements}

My immense gratitude to my Queens for their understanding, love, and support. I thank immensely my Mentor-and great Promoter of Biomed. Science: Akunne Gilbert O. Ofodile, a good Brother and a good Onitsha man., I am very grateful to ONOWU Chike Ofodile (SAN) for the good things he did for the Ofodile-Ezeugo Family, and for the entire Onitsha people, the whole Nigeria and the world. In this continuum, I wish also to express my immense thanks to one of the greatest, good and humble Lady, I have ever known Madam Nwanyima Ofodile (Nne Oseloka ) for contributing enormously to the core of happiness, love, unity and stability, and peace in Ofodile Ezeugo Family. Nwakaibie Gladstone N. Ofodile is also highly acknowledged for his unique and great contribution leading to restoration and strengthening of peace, happiness, and unity to Ofodile Ezeugo Family. I immensely thank Ezenwayi J. Ofodile, a great Woman, for contributing in various specific ways to happiness, peace and stability in Ofodile Ezeugo Family, Madam Ogbogu (Nne Maria), Madam Emodi (Nne Gab.), Madam N. Njoteh, Madam Grace Ofodile (Nne Achike), Madam Christie Ofodile (Nne Odi), Madam Lucy Ogbua (Nne IK.) and Madam Agnes N. Ofodile (Nne Ikem), all great Women and great Mothers are gratefully acknowledged for contributing in a variety of significant ways to peace, progress, happiness, love, and stability in the Ofodile Ezeugo Family. Additionally, in this context: Okom Ofodile would like to take this opportunity to thank immensely the King of Onitsha (Anambra State, Nigeria): Igwe ACHEBE (The King of Onitsha) for contributing highly to stability and happiness in Ofodile Family. I also acknowledge two very great Women: Ma Mgbeogikwe Anyanna (Ma Ocha), and Ma Mrs. Nnoruka (Nne Joe.) for contributing significantly in various ways to the happiness, stability, peace and unity in the Ofodile Ezeugo Family. Very importantly, my immense gratitude to Akunwafor Raymond Ogbogu Ofodile (Sumgbe) for all the good things he did and, contributed to the existence, happiness, unity, love, and stability in the Ezeugo Ofodile Family. I apologize to the authors whose work I could not cite because of the limit on the number of references and therefore mostly cited, sort of, overview articles. Immense thanks to Mr. Burghard Grossmann (the System Administrator, university library, Charite Virchow Campus, Universitätsmediz in Berlin, Germany) for his excellent technical assistance during the preparation of this manuscript. Okom Ofodile thanks Franz Theuring (Dr. Theuring) for his collegiality.

\section{Financial support}

This study was partly supported by Charite' Universitätsmedizin Berlin, Germany.

\section{References}

1. Kostner GM, Avogaro P, Cazzolato G, Marth E, Bittolobon G (1981) LP(a) and the risk for myocardial infarction. Atherosclerosis 38: 51-61.

2. Dahlen G (1988) Lipoprotein (a) in relation to atherosclerotic disease, In: Recen Aspects of diagnosis and treatment of lipoprotein disorders. K Widhalm, H Naito, eds. New York: A. Liss Inc 27-36.

3. Hoefler G, Harnoncourt F, Paschke E, Mirtl W, Pfeiffer KH, et al (1988) Lipoprotein $\mathrm{Lp}$ (a) a risk factor for myocardial infarct. Arteriosclerosis 8: 398-401. [Crossref]

4. Rubinsztein DC, Codogno P, Levine B (2012) Autophagy modulation as potential target for diverse diseases. Nat Rev Drug Discov 11: 709-730. [Crossref]

5. Brest P, Corcelle EA, Cesaro A, Chargui A, Belaid A, et al. (2010) Autophagy and the Crohns'disease: At the Crossroads of infection, Inflammation, Immunity and Cancer. Curr Mol Med 10: 486-502. [Crossref]

6. Singh R., Kauschik S, Wang Y, Xiang Y, Novak I, et al. (2009) Autophagy regulates Lipid Metabolism. Nature 458: 1131-1135. [Crossref]

7. Singh R, Cuervo AM (2011) Autophagy in the Cell energy Balance. Cell Metab 13 495-504. [Crossref]

8. Johansen JS (2006) Studies on serum YKL-40 and biomarker in diseases with inflammation, tissue remodelling, fibrosis and Cancer. Dan Med Bull 53: 172-209. [Crossref]

9. Lee Chun Geun, Da Silva CA, Dela Cruz CS, Ahangari F, Ma B, et al. (2011) Role of Chitin and Chitinase/Chitinase-like Proteins in Inflammation, Tissue Remodeling, and Injury. Annu Rev Physiol 73: 479-501. [Crossref]

10. Renkema GH, Boot RG, Muijsers AO, Donker-Koopman WE, Aerts JM (1995) Purification and characterization of human chitotriosidase, a novel member of the chitinase family of proteins. J Biol Chem 270: 2198-202. [Crossref]

11. Hakala BE, White C, Recklies AD (1993) Human Cartilage Glycoprotein 39, a major secretory product of articular chondrocytes and synovial cells, is a mammalian member of a chitinase protein family. J Biol Chem 268: 25803-25810. [Crossref]

12. Kjoergaard AD, Bgesen SE, Johansen JS, Nordestgaard BG (2010) Elevated levels of YKL-40 and Ischemic stroke. Annu Neurol 68: 627-680. [Crossref]

13. Bilim O, Takeishi Y, Kitahara T, Ishino M, Sasaki T, et al. (2010) Serum YKL-40 predicts advanced clinical outcome in patients with chronic heart failure. J Card Fail 16: 873-879. [Crossref]

14. Chang NC, Hung SI, Hwa KY, Kato I, Chen JE, et al. (2001) A macrophage protein, $\mathrm{Ym} 1$, transiently expressed during inflammation is a novel mammalian lectin. $J$ Biol Chem 276: 17497-506. [Crossref]

15. Calabrese EJ, Baldwin LA (2002) Defining Hormesis. Hum Exp Toxicol 21: 91-97. [Crossref]

16. Thorp E, Tobas I (2009) Mechanisms and consequences in advanced atherosclerosis. $J$ Leukoc Biol 86: 1089-95. [Crossref]

17. Virmani R, Burke AP, Kolodgie FD, Farb A (2002) Vulnerable plague: the pathology of unstable coronary lesions. J Interv Cardiol 15: 439-46. [Crossref]

18. Rajasekeran NS, Christians ES, Yan LJ, Taylor RP, Orosz A, et al. (2007) Human alpha/beta -cystallin mutation causes oxidative stress and protein aggregation leading to cardiomyopathy in mice. Cell 130: 427-39. [Crossref]

19. Neafsey PJ (1990) Longevity, Hormesis A Review. Mech Ageing Dev 51: 1-31 [Crossref]

20. Kristal BS, Yu BP (1994) Aging and its modulation by Dietary restriction. In Modulation of Aging process by Dietary Restriction. BP Yu, Ed: 1-36, CRC Press. Boca Raton, FL. 
OFODILE ONFC (2015) Lipopritein (a), a principle culprit: Relevance to pathobiology of hypertension, ischemic strokes and implications for novel therapeutic targets, a new puzzle found

21. Yu BP (2006) Why Calorie Restriction works for human longevity. Biogerontology 7(3): 179-82. [Crossref]

22. Rezzi S, Martin FP, Shanmhganayagam D, Colman RI, Nicholson IK, et al. (2009) Metabolic shift due to long-term calorie restriction revealed in nonhuman primates. Exp Gerontol 44: 356-62. [Crossref]
23. Turturro A, Duffy P, Hart RW (1993) Modulation of Toxicity by diet and dietary macronutrient restriction. Mutat Res 295: 151-164. [Crossref]

24. Fontana L, Meyer TE, Klein S, Holloszy JO (2004) Long term calorie restriction is highly effective in reducing the risk for atherosclerosis in humans. Proc Natl Acad Sci US A 101: 17: 6659-6663. [Crossref]

25. Barja G (2002) Quantitative measurement of $\mathrm{H} 202$ generation in isolated mitochondria. J Bioenerg Biomembran 34: 227-33. [Crossref]

Copyright: $\bigcirc 2015$ Ofodile ONFC. This is an open-access article distributed under the terms of the Creative Commons Attribution License, which permits unrestricted use, distribution, and reproduction in any medium, provided the original author and source are credited. 